ANL-5121

Chemistry - General

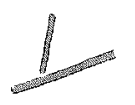

ARGONNE NATIONAL LABORATORY

P. O. Box 299

Lemont, Illinois

ENGINEERING PROPERTIES OF DIPHENYL

by

Kermit Anderson

REACTOR ENGINEERING DIVISION

August 11, 1953

Operated by The University of Chicago

under

Contract $W-31-109-e n g-38$

Gis? 


\section{DISCLAIMER}

This report was prepared as an account of work sponsored by an agency of the United States Government. Neither the United States Government nor any agency Thereof, nor any of their employees, makes any warranty, express or implied, or assumes any legal liability or responsibility for the accuracy, completeness, or usefulness of any information, apparatus, product, or process disclosed, or represents that its use would not infringe privately owned rights. Reference herein to any specific commercial product, process, or service by trade name, trademark, manufacturer, or otherwise does not necessarily constitute or imply its endorsement, recommendation, or favoring by the United States Government or any agency thereof. The views and opinions of authors expressed herein do not necessarily state or reflect those of the United States Government or any agency thereof. 


\section{DISCLAIMER}

Portions of this document may be illegible in electronic image products. Images are produced from the best available original document. 


\section{FOREWORD}

The main body of the physical data on pure dipheny1 is the property of the Monsanto Chemical Company and has been published by them in various technical reports and sales literature.

Data on vapor pressure, enthalpy and densities of liquid diphenyl and its saturated vapor were made available by members of the Monsanto Chemical Company research staff at Anniston, Alabama. This was also the source of information on the viscosity of diphenyl as a function of temperature, the Mollier diagram and the temperature entropy diagram.

The data on the thermal conductivity of Dowtherm A was furnished by the Dow Chemical Company.

The phase-rule diagrams for diphenyl and the diphenyl benzenes and for mixtures of diphenyl and its pyrolysis prod ucts along with viscosities of three mixtures were determined at Argonne. 
TABLE OF CONTENTS

Page

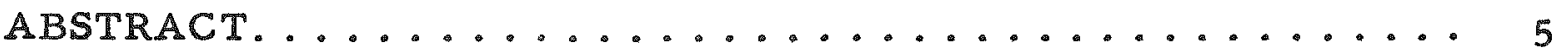

I. PHYSICAL PROPERTIES OF DIPHENYL ........... 5

1. Miscellaneous Physical Data on Diphenyl........ 5

2. Thermodynamic Properties of Diphenyl ......... 5

3. Mollier Diagram .................... 12

4. Temperature-Entropy Diagram for Diphenyl....... 12

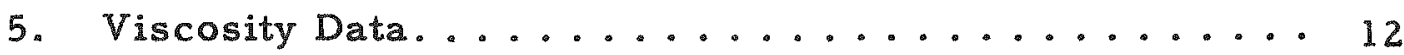

6. Thermal Conductivity .................. 12

7. Coefficients of Heat Transfer.............. 13

II. DATA ON MIXTURES OF DIPHENYL WITH OTHER MATERIAL . 13

1. Mixtures of Diphenyl with o-Diphenylbenzene ...... 14

2. Mixtures of Diphenyl with m-Diphenylbenzene....... 14

3. Mixtures of Diphenyl with $\mathrm{p}$-Diphenylbenzene ...... 14

4. Mixtures of Diphenyl with its Pyrolysis Products ..... 14 


\section{LIST OF FIGURES}

Figure

Title

Page

1 Mollier Diagram for Dipheny1 .............. 16

2 Temperature Entropy Diagram.............. 17

3 Calculated Thermal Conductivity of Dowtherm A ...... 18

4 Liquid Film Coefficient for Dowtherm Inside Pipes ...... 19

5 Melting Points for the System Diphenyl - o-Diphenylbenzene. . 20

6 Kinematic Viscosity of Mixtures of Diphenyl with

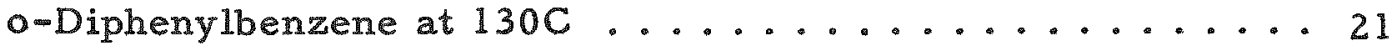

7 Melting Points for the System Diphenyl - m-Diphenylbenzene. 22

8 Kinematic Viscosity of Mixtures of Diphenyl with m-Diphenylbenzene at $130 \mathrm{C} \ldots \ldots \ldots 23$

9 Melting Points for the System Dipheny1 - p-Diphenylbenzene . 24

10 Kinematic Viscosity of Mixtures of Diphenyl with p-Diphenylbenzene at $130 \mathrm{C} \ldots \ldots \ldots \ldots$

11 Melting Points for the System Diphenyl-Tax ........ 26

12 Kinematic Viscosity of Mixtures of Diphenyl with Pyrolytic Tars at $130 \mathrm{C} \ldots \ldots \ldots \ldots . \ldots \ldots 27$

\section{LIST OF TABLES}

Table

Title

$\underline{\text { Page }}$

I Miscellaneous Properties of Diphenyl ............ 6

II Thermal Properties of Diphenyl .............. 7

III Viscosity of Diphenyl at Various Temperatures........ 12

IV Approximate Analysis of Pyrolytic Materials ........ 15 


\title{
ENGINEERING PROPERTIES OF DIPHENYL
}

by

Kermit Anderson

\begin{abstract}
Data collected from the literature on the vapor pressure, enthalpy, liquid density, and vapor density of pure diphenyl are presented. A Mollier diagram, a temperature entropy diagram, and data on the viscosity of diphenyl as a function of temperature are also presented.

Data on the melting points of several binary systems containing diphenyl and for complex mixtures of diphenyl and its pyrolysis products are presented graphically. Graphs of viscosity versus composition at $130 \mathrm{C}$ for the systems are also presented.
\end{abstract}

\section{PHYSICAL PROPERTIES OF DIPHENYL}

These data were obtained by the research staff of the Monsanto Chemical Company and of the Dow Chemical Company. No discussion of the methods used in obtaining the data presented herewith will be given. Original sources can be consulted for the methods used in obtaining the data.

\section{Miscellaneous Physical Data on Diphenyl}

Table I consists of a collection of miscellaneous data on diphenyl. Part of these items were furnished by the Dow Chemical Company of Midland, Michigan, the remainder being taken from standard chemical handbooks.

\section{Thermodynamic Properties of Dipheny1}

The thermal properties of diphenyl are quoted in Table II from data compiled by the technical staff of the Monsanto Chemical Company. ${ }^{1}$

1 The following is quoted from the acknowledgement in the Monsanto Chemical Company's technical files at Anniston, Alabama.

${ }^{66}$ In collecting this data several different sources have been consulted, and we wish especially to acknowledge the work done by the following:

Professor J.M. Cork and Mr. D. A. Wilbur of the University of 
Table I

MISCELLANEOUS PROPERTIES OF DIPHENYL

\begin{tabular}{|l|c|}
\hline Molecular Weight, gms/gm mol & $154 . \overline{2}$ \\
Melting point, ${ }^{\circ} \mathrm{C}$ & 69.4 \\
Specific gravity at $75.4^{\circ} \mathrm{C}$, gms/cc & 0.991 \\
Lbs/gal at $75^{\circ} \mathrm{C}$ & 8.27 \\
Flash point (Cleveland Open Cup), ${ }^{\circ} \mathrm{C}$ & 106 \\
Fire Point (Cleveland Open Cup), ${ }^{\circ} \mathrm{C}$ & 124 \\
Specific heat at boiling point, Btu/(1b)(F) & 0.60 \\
Latent heat of vaporization at boiling point, Btu/1b & 136.5 \\
Heat of Fusion, Btu/1b & 177.8 \\
Solubility at $25^{\circ} \mathrm{C}$, gms/100 gms solvent & \\
Acetone & 95 \\
Benzene & 95 \\
Carbon Tetrachloride & 143 \\
Ether & 63 \\
N-Leptane & 19 \\
Methanol & 7 \\
Water & 0.1 \\
\hline
\end{tabular}

Michigan for work done on vapor pressure, latent heat, and densities of both the vapor and liquid diphenyl.

Laboratory of Applied Chemistry, Massachusetts Institute of Technology, for work done on specific heat of the liquid which work was done by Messrs. L.W. Cummings, F.W. Stones, M. A. Volante, under the direction of Messrs. H. O. Forrest and Elmer W. Brugmann.

Mr.R. O. Fowler of the University of Michigan, for work on specific heat of the liquid at temperatures below the boiling point.

Messrs. John Chipman and S.R. Peltier, Georgia School of Technology, for therr article appearing in Industrial and Engineering Chemistry, Volume 21, page 1106, (1929) relating to vapor pressures.

Dr.W.L. McCabe, University of Michigan, and associates for work done in collecting these data and preparing them for publication.

Professor W. L. Badger, Swenson Evaporator Company, for his cooperation in the preparation of these tables and for the timely and helpful suggestions which he has offered." 
Table II

THERMAL PROPERTIES OF DIPHENYL

(Courtesy Monsanto Chemical Company)

\begin{tabular}{|c|c|c|c|c|c|c|c|c|}
\hline \multicolumn{2}{|c|}{ Temperature } & \multicolumn{2}{|c|}{ Pressure } & \multicolumn{3}{|c|}{$\begin{array}{l}\text { Heat Content } \\
\text { Btu/lb }\end{array}$} & \multicolumn{2}{|c|}{$\begin{array}{l}\text { Density } \\
\mathrm{lb} / \mathrm{cu} \mathrm{ft}\end{array}$} \\
\hline$F$ & C & $\begin{array}{l}\mathrm{Hg} \mathrm{Vac} . \\
\text { In. }\end{array}$ & $\begin{array}{l}\text { psi, } \\
\text { abs. }\end{array}$ & Liquid & Latent & Total & Liquid & Vapor \\
\hline 156.6 & 69.2 & 29.9 & 0.015 & 0.0 & 190.9 & 190.9 & - & - \\
\hline 160 & 71.1 & 29.9 & 0.017 & 1.4 & 189.0 & 190.4 & - & - \\
\hline 165 & 73.9 & 29.9 & 0.020 & 3.4 & 187.5 & 190.9 & - & - \\
\hline 170 & 76.7 & 29.9 & 0.024 & 5.4 & 185.5 & 190.9 & - & - \\
\hline 175 & 79.4 & 29.9 & 0.028 & 7.4 & 183.5 & 190.9 & - & $=$ \\
\hline 180 & 82.2 & 29.9 & 0.033 & 9.5 & 182.0 & 191.5 & 61.36 & 0.00058 \\
\hline 185 & 85.0 & 29.8 & 0.038 & 11.5 & 180.0 & 191.5 & 61.24 & 0.00067 \\
\hline 190 & 87.8 & 29.8 & 0.044 & 13.6 & 178.5 & 192.1 & 61.11 & 0.00078 \\
\hline 195 & 90.7 & 29.8 & 0.052 & 15.6 & 176.5 & 192.1 & 60.98 & 0.00092 \\
\hline 200 & 93.3 & 29.8 & 0.060 & 17.7 & 175.0 & 192.7 & 60.85 & 0.00108 \\
\hline 205 & 96.1 & 29.8 & 0.069 & 19.8 & 173.0 & 192.8 & 60.72 & 0.00127 \\
\hline 210 & 98.9 & 29.8 & 0.080 & 21.9 & 171.5 & 193.4 & 60.59 & 0.00147 \\
\hline 215 & 101.7 & 29.7 & 0.092 & 24.0 & 170.0 & 194.0 & 60.45 & 0.00169 \\
\hline 220 & 104.4 & 29.7 & 0.105 & 26.1 & 168.5 & 194.6 & 60.31 & 0.00190 \\
\hline 225 & 107.2 & 29.7 & 0.120 & 28.3 & 167.0 & 195.3 & 60.17 & 0.00214 \\
\hline 230 & 110.0 & 29.6 & 0.137 & 30.4 & 166.0 & 196.4 & 60.03 & 0.00242 \\
\hline 235 & 112.8 & 29.6 & 0.156 & 32.6 & 164.5 & 197.1 & 59.89 & 0.00273 \\
\hline 240 & 115.6 & 29.6 & 0.177 & 34.8 & 163.5 & 198.3 & 59.75 & 0.00317 \\
\hline 245 & 118.3 & 29.5 & 0.201 & 36.9 & 162.5 & 199.4 & 59.60 & 0.00359 \\
\hline 250 & 121.1 & 29.5 & 0.227 & 39.1 & 161.5 & 200.6 & 59.46 & 0.0041 \\
\hline 255 & 123.9 & 29.4 & 0.256 & 41.3 & 160.5 & 201.8 & 59.32 & 0.0047 \\
\hline 260 & 126.7 & 29.3 & 0.289 & 43.6 & 159.5 & 203.1 & 59.18 & 0.0053 \\
\hline 265 & 129.4 & 29.3 & 0.325 & 45.8 & 158.5 & 204.3 & 59.04 & 0.0059 \\
\hline 270 & 132.2 & 29.2 & 0.365 & 48.1 & 157.5 & 205.6 & 58.90 & 0.0065 \\
\hline 275 & 135.0 & 29.1 & 0.408 & 50.3 & 157.0 & 207.3 & 58.76 & 0.0071 \\
\hline 280 & 137.8 & 2 & 0.457 & 52.6 & 156.5 & 209.1 & 58.62 & 0.0078 \\
\hline 285 & 140.6 & 28.9 & 0.509 & 54.9 & 155.5 & 210.4 & 58.48 & 0.0086 \\
\hline 290 & 143.3 & 28.8 & 0.568 & 57.2 & 155.0 & 212.2 & 58.35 & 0.0095 \\
\hline 295 & 146.1 & 28.6 & 0.631 & 59.6 & 154.5 & 214.1 & 58.21 & 0.0105 \\
\hline 300 & 148.7 & 28.5 & 0.701 & 61.9 & 154.0 & 215.9 & 58.08 & 0.0117 \\
\hline 305 & 151.7 & 28.3 & 0.776 & 64.3 & 153.5 & 217.8 & 57.94 & 0.0129 \\
\hline 310 & 154.4 & 28.2 & 0.860 & 66.6 & 153.0 & 219.6 & 57.80 & 0.0142 \\
\hline 315 & 157.2 & 28.0 & 0.949 & 69.0 & 152.5 & 221.5 & 57.66 & 0.0156 \\
\hline 320 & 160.0 & 27.8 & 1.050 & 71.4 & 152.0 & 223.4 & 57.52 & 0.0170 \\
\hline 325 & 162.8 & 27.6 & 1.150 & 73.9 & 151.5 & 225.4 & 57.36 & 0.0187 \\
\hline
\end{tabular}


Table II (Continued)

\begin{tabular}{|c|c|c|c|c|c|c|c|c|}
\hline \multicolumn{2}{|c|}{ T'emperature } & \multicolumn{2}{|c|}{ Pressure } & \multicolumn{3}{|c|}{$\begin{array}{l}\text { Heat Content } \\
\text { Btu/1b }\end{array}$} & \multicolumn{2}{|c|}{$\begin{array}{l}\text { Density } \\
\mathrm{lb} / \mathrm{cu} \mathrm{ft}\end{array}$} \\
\hline$F$ & $\mathrm{C}$ & $\underset{\text { In. }}{\mathrm{Hg} \text { Vac. }}$ & $\begin{array}{l}\text { psi, } \\
\text { abs. }\end{array}$ & Liquid & Latent & Total & Liquid & Vapor \\
\hline 330 & 165.6 & 27.3 & 1.27 & 76.3 & 151.5 & 227.8 & 57.22 & 0.0203 \\
\hline 335 & 168.3 & 27.1 & 1.39 & 78.8 & 151.0 & 229.8 & 57.08 & 0.0219 \\
\hline 340 & 171.1 & 26.8 & 1.53 & 81.3 & 151.0 & 232.3 & 56.93 & 0.0239 \\
\hline 345 & 173.9 & 26.5 & 1.67 & 83.8 & 150.5 & 234.3 & 56.79 & 0.0260 \\
\hline 350 & 176.7 & 26.2 & 1.83 & 86.3 & 150.0 & 236.3 & 56.65 & 0.0284 \\
\hline 355 & 179.4 & 25.8 & 2.00 & 88.8 & 150.0 & 238.8 & 56.50 & 0.0311 \\
\hline 360 & 182.2 & 25.5 & 2.18 & 91.4 & 149.5 & 240.9 & 56.36 & 0.0336 \\
\hline 365 & 185.0 & 25.1 & 2.38 & 94.0 & 149.5 & 243.5 & 56.22 & 0.0366 \\
\hline 370 & 187.8 & 24.7 & 2.59 & 96.5 & 149.0 & 245.5 & 56.08 & 0.0396 \\
\hline 375 & 190.6 & 24.2 & 2.81 & 99.2 & 149.0 & 248.2 & 55.94 & 0.0427 \\
\hline 380 & 193.3 & 23.7 & 3.06 & 101.8 & 149.0 & 250.8 & 55.80 & 0.0464 \\
\hline 385 & 196.1 & 23.2 & 3.31 & 104.5 & 148.5 & 253.0 & 55.67 & 0.0497 \\
\hline 390 & 198.9 & 22.6 & 3.59 & 107.1 & 148.5 & 255.6 & 55.53 & 0.0536 \\
\hline 395 & 201.7 & 22.0 & 3.88 & 109.9 & 148.0 & 257.9 & 55.39 & 0.0575 \\
\hline 400 & 204.4 & 21.4 & 4.20 & 112.6 & 148.0 & 260.6 & 55.24 & 0.0616 \\
\hline 405 & 207.2 & 20.7 & 4.53 & 115.3 & 148.0 & 263.3 & 55.10 & 0.0659 \\
\hline 410 & 210.0 & 20.0 & 4.89 & 118.1 & 147.5 & 265.6 & 54.96 & 0.0704 \\
\hline 415 & 212.8 & 19.2 & 5.27 & 120.9 & 147.5 & 268.4 & 54.81 & 0.0753 \\
\hline 420 & 215.6 & 18.4 & 5.67 & 123.7 & 147.0 & 270.7 & 54.67 & 0.0804 \\
\hline 425 & 218.3 & 17.5 & 6.09 & 126.5 & 147.0 & 273.5 & 54.52 & 0.0859 \\
\hline 430 & 221.1 & 16.6 & 6.55 & 129.3 & 146.0 & 275.3 & 54.37 & 0.0918 \\
\hline 435 & 223.9 & 15.6 & 7.03 & 132.2 & 145.5 & 277.7 & 54.23 & 0.0982 \\
\hline 440 & 226.7 & 14.6 & 7.54 & 135.1 & 145.0 & 280.1 & 54.09 & 0.1050 \\
\hline 445 & 229.4 & 13.5 & 8.07 & 138.0 & 144.0 & 282.0 & 53.95 & 0.1120 \\
\hline 450 & 232.2 & 12.3 & 8.64 & 140.9 & 143.5 & 284.4 & 53.81 & 0.1220 \\
\hline 455 & 235.0 & 11.1 & 9.24 & 143.9 & 142.5 & 286.4 & 53.67 & 0.1310 \\
\hline 460 & 237.8 & 9.8 & 9.87 & 146.8 & 142.0 & 288.8 & 53.53 & 0.1410 \\
\hline 465 & 240.6 & 8.4 & 10.50 & 149.8 & 141.0 & 290.8 & 53.38 & 0.1510 \\
\hline 470 & 243.3 & 7.0 & 11.30 & 152.8 & 140.5 & 293.3 & 53.24 & 0.1610 \\
\hline 475 & 246.1 & 5.5 & 12.00 & 155.8 & 139.5 & 295.3 & 53.09 & 0.1700 \\
\hline 480 & 248.9 & 4.0 & 12.80 & 158.8 & 138.5 & 297.3 & 52.93 & 0.1800 \\
\hline 485 & 251.7 & 2.3 & 13.60 & 161.8 & 137.5 & 299.3 & 52.79 & 0.1920 \\
\hline 490 & 254.4 & 0.5 & 14.40 & 164.9 & 136.5 & 301.4 & 52.64 & 0.2050 \\
\hline 491.5 & 255.3 & 0.0 & 14.70 & 165.8 & 136.5 & 302.3 & 52.59 & 0.2100 \\
\hline
\end{tabular}


Table II (Continued)

\begin{tabular}{|c|c|c|c|c|c|c|c|c|}
\hline \multicolumn{2}{|c|}{ Temperature } & \multicolumn{2}{|c|}{$\begin{array}{c}\text { Pressure } \\
\text { psi }\end{array}$} & \multicolumn{3}{|c|}{$\begin{array}{c}\text { Heat Content } \\
\text { Btu/1b }\end{array}$} & \multicolumn{2}{|c|}{$\begin{array}{l}\text { Density } \\
\mathrm{lb} / \mathrm{cu} \mathrm{ft}\end{array}$} \\
\hline $\mathbf{F}$ & C & Gage & Abs. & Liquid & Latent & Total & Liquid & Vapor \\
\hline 491.5 & 255.3 & 0.0 & 14.7 & 165.8 & 136.5 & 302.3 & 52.59 & 0.210 \\
\hline 495 & 257.2 & 0.6 & 15.3 & 168.0 & 135.5 & 303.5 & 52.48 & 0.220 \\
\hline 500 & 260.0 & 1.6 & 16.3 & 171.0 & 135.0 & 306.0 & 52.32 & 0.236 \\
\hline 505 & 262.8 & 2.6 & 17.3 & 174.1 & 134.0 & 308.1 & 52.16 & 0.253 \\
\hline 510 & 265.6 & 3.6 & 18.3 & 177.2 & 133.0 & 310.2 & 52.01 & 0.271 \\
\hline 515 & 268.3 & 4.7 & 19.4 & 180.4 & 132.0 & 312.4 & 51.85 & 0.290 \\
\hline 520 & 271.1 & 5.9 & 20.6 & 183.5 & 131.0 & 314.5 & 51.69 & 0.310 \\
\hline 525 & 273.9 & 7.0 & 21.7 & 186.6 & 130.5 & 317.1 & 51.53 & 0.330 \\
\hline 530 & 276.7 & 8.3 & 23.0 & 189.8 & 129.5 & 319.3 & 51.37 & 0.352 \\
\hline 535 & 279.4 & 9.6 & 24.3 & 192.9 & 128.5 & 321.4 & 51.21 & 0.373 \\
\hline 540 & 282.2 & 11.0 & 25.7 & 196.1 & 127.5 & 323.6 & 51.05 & 0.395 \\
\hline 545 & 285.0 & 12.4 & 27.1 & 199.3 & 127.0 & 326.3 & 50.89 & 0.420 \\
\hline 550 & 287.8 & 13.8 & 28.5 & 202.5 & 126.0 & 328.5 & 50.73 & 0.445 \\
\hline 555 & 290.6 & 15.4 & 30.1 & 205.7 & 125.0 & 330.7 & 50.57 & 0.470 \\
\hline 560 & 293.3 & 17.0 & 31.7 & 208.9 & 124.5 & 333.4 & 50.41 & 0.495 \\
\hline 565 & 296.1 & 18.6 & 33.3 & 212.1 & 123.5 & 335.6 & 50.24 & 0.525 \\
\hline 570 & 298.9 & 20.4 & 35.1 & 215.3 & 122.5 & 337.8 & 50.08 & 0.555 \\
\hline 575 & 301.6 & 22.2 & 36.9 & 218.6 & 121.5 & 340.1 & 49.90 & 0.585 \\
\hline 580 & 304.4 & 24.1 & 38.8 & 221.8 & 121.0 & 342.8 & 49.73 & 0.615 \\
\hline 585 & 307.2 & 26.0 & 40.7 & 225.1 & 120.0 & 345.1 & 49.56 & 0.650 \\
\hline 590 & 310.0 & 28.1 & 42.8 & 228.3 & 119.0 & 347.3 & 49.39 & 0.680 \\
\hline 595 & 312.8 & 30.1 & 44.8 & 231.6 & 118.0 & 349.6 & 49.22 & 0.715 \\
\hline 600 & 315.6 & 32.3 & 47.0 & 234.8 & 117.0 & 351.8 & 49.04 & 0.750 \\
\hline 605 & 318.3 & 34.6 & 49.3 & 238.1 & 116.0 & 354.1 & 48.87 & 0.790 \\
\hline 610 & 321.1 & 36.9 & 51.6 & 241.4 & 115.5 & 356.9 & 48.70 & 0.830 \\
\hline 615 & 323.9 & 39.3 & 54.0 & 244.7 & 114.5 & 359.2 & 48.52 & 0.865 \\
\hline 620 & 326.7 & 41.9 & 56.6 & 248.0 & 114.0 & 362.0 & 48.35 & 0.910 \\
\hline 625 & 329.4 & 44.5 & 59.2 & 251.3 & 113.0 & 364.3 & 48.17 & 0.950 \\
\hline 630 & 332.2 & 47.2 & 61.9 & 254.6 & 112.5 & 367.1 & 47.99 & 0.995 \\
\hline 635 & 335.0 & 49.9 & 64.6 & 257.9 & 111.5 & 369.4 & 47.82 & 1.040 \\
\hline
\end{tabular}


Table II (Continued)

\begin{tabular}{|c|c|c|c|c|c|c|c|c|}
\hline \multicolumn{2}{|c|}{ Temperature } & \multicolumn{2}{|c|}{$\begin{array}{l}\text { Pressure } \\
\text { psi }\end{array}$} & \multicolumn{3}{|c|}{$\begin{array}{c}\text { Heat Content } \\
\text { Btu } / 1 \mathrm{~b}\end{array}$} & \multicolumn{2}{|c|}{$\begin{array}{l}\text { Density } \\
1 \mathrm{~b} / \mathrm{cu} \mathrm{ft}\end{array}$} \\
\hline$F$ & $\mathrm{C}$ & Gage & Abs. & Liquid & Latent & Total & Liquid & Vapor \\
\hline 640 & 337.8 & 52.8 & 67.5 & 261.2 & 111.0 & 372.2 & 47.64 & 1.085 \\
\hline 645 & 340.6 & 55.8 & 70.5 & 264.6 & 110.0 & 374.6 & 47.48 & 1.135 \\
\hline 650 & 343.3 & 58.9 & 73.6 & 267.9 & 109.5 & 377.4 & 47.30 & 1.185 \\
\hline 655 & 346.1 & 62.0 & 76.7 & 271.2 & 109.0 & 380.2 & 47.12 & 1.235 \\
\hline 660 & 348.9 & 65.3 & 80.0 & 274.6 & 108.5 & 383.1 & 46.95 & 1.285 \\
\hline 665 & 351.7 & 68.7 & 83.4 & 277.9 & 107.5 & 385.4 & 46.77 & 1.340 \\
\hline 670 & 354.4 & 72.2 & 86.9 & 281.3 & 107.0 & 388.3 & 46.59 & 1.395 \\
\hline 675 & 357.2 & 75.8 & 90.5 & 284.6 & 106.5 & 391.1 & 46.40 & 1.450 \\
\hline 680 & 360.0 & 79.5 & 94.2 & 288.0 & 105.5 & 393.5 & 46.20 & 1.510 \\
\hline 685 & 362.8 & 83.3 & 98.0 & 291.4 & 105.0 & 396.4 & 45.97 & 1.565 \\
\hline 690 & 365.6 & 87.2 & 101.9 & 294.7 & 104.0 & 398.7 & 45.77 & 1.620 \\
\hline 695 & 368.3 & 91.2 & 105.9 & 298.1 & 103.5 & 401.6 & 45.58 & 1.675 \\
\hline 700 & 371.1 & 95.4 & 110.1 & 301.5 & 103.0 & 404.5 & 45.38 & 1.730 \\
\hline 705 & 373.9 & 99.7 & 114.4 & 304.8 & 102.5 & 407.3 & 45.19 & 1.785 \\
\hline 710 & 376.7 & 104.1 & 118.8 & 308.2 & 102.0 & 410.2 & 44.98 & 1.840 \\
\hline 715 & 379.4 & 108.6 & 123.3 & 311.6 & 101.0 & 412.6 & 44.77 & 1.900 \\
\hline 720 & 382.2 & 113.3 & 128.0 & 315.0 & 100.5 & 415.5 & 44.57 & 1.965 \\
\hline 725 & 385.0 & 118.0 & 132.7 & 318.4 & 100.0 & 418.4 & 44.36 & 2.030 \\
\hline 730 & 387.8 & 122.9 & 137.6 & 321.8 & 99.0 & 420.8 & 44.15 & 2.100 \\
\hline 735 & 390.6 & 128.0 & 142.7 & 325.2 & 98.5 & 423.7 & 43.93 & 2.170 \\
\hline 740 & 393.3 & 133.3 & 148.0 & 328.6 & 98.0 & 426.6 & 43.72 & 2.245 \\
\hline 745 & 396.1 & 138.6 & 153.3 & 332.0 & 97.0 & 429.0 & 43.51 & 2.320 \\
\hline 750 & 398.9 & 143.9 & 158.6 & 335.4 & 96.5 & 431.9 & 43.28 & 2.400 \\
\hline 755 & 401.7 & 149.5 & 164.2 & 338.8 & 96.0 & 434.8 & 43.05 & 2.480 \\
\hline 760 & 404.4 & 155.2 & 169.9 & 342.2 & 96.0 & 438.2 & 42.82 & 2.565 \\
\hline 765 & 407.2 & 161.0 & 175.7 & 345.6 & 95.5 & 441.1 & 42.58 & 2.655 \\
\hline 770 & 410.0 & 167.1 & 181.8 & 349.0 & 95.0 & 444.0 & 42.35 & 2.750 \\
\hline 775 & 412.8 & 173.3 & 188.0 & 352.4 & 94.5 & 446.9 & 42.11 & 2.845 \\
\hline 780 & 415.6 & 179.7 & 194.4 & 355.9 & 94.0 & 449.9 & 41.87 & 2.945 \\
\hline 785 & 418.3 & 186.2 & 200.9 & 359.3 & 93.5 & 452.8 & 41.64 & 3.045 \\
\hline 790 & 421.1 & 193.0 & 207.7 & 362.7 & 93.0 & 445.7 & 41.39 & 3.145 \\
\hline 795 & 423.9 & 200.0 & 214.7 & 366.1 & 92.0 & 458.1 & 41.14 & 3.250 \\
\hline 800 & 426.7 & 207.1 & 221.8 & 369.6 & 91.5 & 461.1 & 40.89 & 3.355 \\
\hline 805 & 429.4 & 214.1 & 228.8 & 373.0 & 90.5 & 463.5 & 40.64 & 3.465 \\
\hline 810 & 432.2 & 221.2 & 235.9 & 376.4 & 90.0 & 466.4 & 40.38 & 3.580 \\
\hline
\end{tabular}


Table II (Continued)

\begin{tabular}{|c|c|c|c|c|c|c|c|c|}
\hline \multicolumn{2}{|c|}{ Temperature } & \multicolumn{2}{|c|}{$\begin{array}{l}\text { Pressure, } \\
\text { psi }\end{array}$} & \multicolumn{3}{|c|}{$\begin{array}{c}\text { Heat Content } \\
\text { Btu/1b }\end{array}$} & \multicolumn{2}{|c|}{$\begin{array}{l}\text { Density } \\
\mathrm{lb} / \mathrm{cu} \mathrm{ft}\end{array}$} \\
\hline$F$ & $\mathrm{C}$ & Gage & Abs. & Liquid & Latent & Total & Liquid & Vapor \\
\hline 815 & 435.0 & 228.6 & 243.3 & 379.9 & 89.0 & 468.9 & 40.12 & 3.700 \\
\hline 820 & 437.8 & 236.3 & 251.0 & 383.3 & 88.5 & 471.8 & 39.84 & 3.825 \\
\hline 825 & 440.8 & 244.2 & 258.9 & 386.7 & 87.5 & 474.2 & 39.56 & 3.955 \\
\hline 830 & 443.3 & 252.2 & 266.9 & 390.1 & 86.5 & 476.6 & 39.28 & 4.090 \\
\hline 835 & 446.1 & 260.3 & 275.0 & 393.6 & 86.0 & 479.6 & 38.99 & 4.230 \\
\hline 840 & 448.9 & 268.6 & 283.3 & 397.0 & 85.0 & 482.0 & 38.69 & 4.375 \\
\hline 845 & 451.7 & 277.3 & 292.0 & 400.5 & 84.0 & 484.5 & 38.40 & 4.530 \\
\hline 850 & 454.4 & 286.2 & 300.9 & 403.9 & 82.5 & 486.4 & 38.10 & 4.690 \\
\hline 855 & 457.2 & 295.1 & 309.8 & 407.4 & 81.5 & 488.9 & 37.79 & 4.860 \\
\hline 860 & 460.0 & 304.2 & 318.9 & 410.8 & 80.0 & 490.8 & 37.48 & 5.040 \\
\hline 865 & 462.8 & 313.5 & 328.2 & 414.2 & 78.5 & 492.7 & 37.17 & 5.225 \\
\hline 870 & 465.6 & 323.1 & 337.8 & 417.7 & 77.0 & 494.7 & 36.84 & 5.420 \\
\hline 875 & 468.3 & 332.9 & 347.6 & 421.1 & 75.5 & 496.6 & 36.49 & 5.625 \\
\hline 880 & 471.1 & 342.9 & 357.6 & 424.6 & 74.0 & 498.6 & 36.14 & 5.845 \\
\hline 885 & 473.9 & 353.1 & 367.8 & 428.0 & 72.5 & 500.5 & 35.79 & 6.070 \\
\hline 890 & 476.7 & 363.5 & 378.2 & 431.5 & 71.0 & 502.5 & 35.41 & 6.320 \\
\hline 895 & 479.4 & 374.1 & 388.8 & 435.0 & 69.5 & 504.5 & 35.01 & 6.570 \\
\hline 900 & 482.2 & 385.0 & 399.7 & 438.4 & 67.5 & 505.9 & 34.58 & 6.850 \\
\hline 905 & 485.0 & 396.1 & 410.8 & 441.9 & 66.0 & 507.9 & 34.14 & 7.140 \\
\hline 910 & 487.8 & 407.5 & 422.2 & 445.3 & 64.0 & 509.3 & 33.68 & 7.455 \\
\hline 915 & 490.6 & 419.0 & 433.7 & 448.8 & 62.0 & 510.8 & 33.16 & 7.790 \\
\hline 920 & 493.3 & 430.9 & 445.6 & 452.2 & 59.5 & 511.7 & 32.61 & 8.145 \\
\hline 925 & 496.1 & 442.9 & 457.6 & 455.7 & 56.5 & 512.2 & 32.02 & 8.520 \\
\hline 930 & 498.9 & 455.2 & 469.9 & 459.1 & 53.5 & .513 .6 & 31.40 & 8.930 \\
\hline 935 & 501.7 & 467.7 & 482.4 & 462.6 & 50.5 & 513.1 & 30.70 & 9.440 \\
\hline 940 & 504.4 & 480.6 & 495.3 & 466.0 & 47.0 & 513.0 & 29.93 & 10.070 \\
\hline 945 & 507.2 & 493.6 & 508.3 & 469.5 & 43.0 & 512.5 & 29.07 & 10.800 \\
\hline 950 & 510.0 & 506.9 & 521.6 & 473.0 & 39.0 & 512.0 & 28.14 & 11.700 \\
\hline 955 & 512.8 & 520.4 & 535.1 & 476.4 & 34.5 & 510.9 & 27.10 & 12.700 \\
\hline 960 & 515.6 & 534.3 & 549.0 & 479.9 & 29.5 & 509.4 & 26.00 & 13.850 \\
\hline 965 & 518.3 & 548.4 & 563.1 & 483.3 & 24.5 & 507.8 & 24.90 & 15.150 \\
\hline 970 & 521.1 & 562.7 & 577.4 & 486.8 & 19.0 & 505.8 & 23.60 & 16.550 \\
\hline 975 & 523.9 & 577.3 & 592.0 & 490.2 & 12.5 & 502.7 & 22.20 & 18.050 \\
\hline 980 & 526.7 & 592.3 & 607.0 & 493.7 & 0.0 & 493.7 & 19.60 & 19.600 \\
\hline
\end{tabular}




\section{Mollier Diagram}

The Mollier Diagram of the diphenyl system is shown in Figure 1.

\section{Temperature-Entropy Diagram for Diphenyl}

The temperature-entropy diagram is shown in Figure 2. A temperature entropy diagram for water is also included for comparison.

\section{Viscosity Data}

The viscosity of diphenyl as a function of temperature is shown in Table III. Data were obtained using Ostwald type viscometers by standard techniques.

\section{Table III}

VISCOSITY OF DIPHENYL AT VARIOUS TEMPERATURES

(Determined by Eugene C. Bingham, Monsanto Chemical Company)

\begin{tabular}{|c|c||c|c|}
\hline Temp, F. & $\begin{array}{c}\text { Viscosity, } \\
\text { Centipoises }\end{array}$ & Temp, F. & $\begin{array}{c}\text { Viscosity, } \\
\text { Centipoises }\end{array}$ \\
\hline 160 & 1.439 & 330 & 0.501 \\
170 & 1.309 & 340 & 0.480 \\
180 & 1.208 & 350 & 0.459 \\
190 & 1.114 & 360 & 0.440 \\
200 & 1.034 & 370 & 0.422 \\
210 & 0.961 & 380 & 0.405 \\
220 & 0.901 & 390 & 0.388 \\
230 & 0.848 & 400 & 0.374 \\
240 & 0.797 & 410 & 0.360 \\
250 & 0.752 & 420 & 0.347 \\
260 & 0.711 & 430 & 0.335 \\
270 & 0.673 & 440 & 0.324 \\
280 & 0.636 & 450 & 0.313 \\
290 & 0.605 & 460 & 0.303 \\
300 & 0.574 & 470 & 0.293 \\
310 & 0.549 & 480 & 0.284 \\
320 & 0.524 & 482 & 0.283 \\
\hline
\end{tabular}

\section{Thermal Conductivity}

Data on the thermal conductivity of diphenyl are not available. It is felt, however, that due to the high diphenyl content and basic similarity 
of Dowtherm A to diphenyl, data for the thermal conductivity of Dowtherm A would lead to no serious errors. Figure 3 shows a plot of these data as well as data for Dowtherm $\mathrm{E}$ as a function of temperature.2

\section{Coefficients of Heat Transfer}

Data are lacking on this property. Figure 4 graphically presents the liquid film coefficient for Dowtherm $A$ inside pipes. In this figure $G^{\prime}=$ mass velocity in $\mathrm{lb} /(\mathrm{sec})\left(\mathrm{ft}^{2}\right), \mathrm{h}=$ film coefficient of heat transfer in $\mathrm{Btu} /(\mathrm{hr})\left(\mathrm{ft}^{2}\right)(\mathrm{F})$, and $\mathrm{D}^{-}=$inside diameter of pipe in in. ${ }^{2}$

\section{DATA ON MIXTURES OF DIPHENYL WITH OTHER MATERIAL}

Since chemical reactions of diphenyl occur under conditions which would be encountered in heat transfer systems, it is of interest to consider the results of mixing some of the more common reaction products of diphenyl with the pure material. In general, the more probable compounds would be the isomeric terphenyls and tetraphenyls, and several alkyl benzenes.

The most significant physical properties of such systems might be their melting points, vapor pressures, and viscosities. Thermal properties are also important, but the se would probably not differ greatly from the same properties for diphenyl if variation in composition of the system is restricted to those ranges where melting points and viscosities are not too greatly changed.

No data are available on the physical properties of mixtures of diphenyl with the various pure alkyl benzenes which might be formed by ring scission in the diphenyl molecule. Neither are data available on the properties of the binary systems consisting of diphenyl and the isomeric tetraphenyls. Preliminary work at Argonne has yielded the data presented below on binary systems of diphenyl and the three isomeric terphenyls and on mixtures of diphenyl with still bottoms from the diphenyl purification stills at the Anniston, Alabama, plant of the Monsanto Chemical Company. The latter tar-like material is considered to be probably quite representative of the complex systems which might result from the pyrolysis or radiolysis of diphenyl.

Melting points of all of these mixtures were determined by conventional methods, mostly by observation of cooling curves run on large samples of originally molten material. Viscosities were determined at $130^{\circ} \mathrm{C}$ using Ostwald pipettes.

2 "Dowtherm for Accurate High Temperature, Low Pressure Heat," The Dow Chemical Company, Midland, Michigan 
1. Mixtures of Diphenyl with o-Diphenylbenzene

Figure 5 shows data on the melting points of the system diphenylo-diphenylbenzene. Viscosities for the same system determined at $130 \mathrm{C}$ are plotted as functions of composition in Figure 6.

2. Mixtures of Diphenyl with m-Diphenylbenzene

Data on melting points for this system are plotted in Figure 7, while the kinematic viscosities at $130 \mathrm{C}$ are plotted against composition in Figure 8.

3. Mixtures of Diphenyl with $\mathrm{p}$-Diphenylbenzene

Data on melting points for this system are shown in Figure 9. Figure 10 gives data on kinematic viscosities for these mixtures only up to a composition of 20 per cent $p$-diphenylbenzene. The melting points for compositions much above this temperature were too high to be liquid at $130 \mathrm{C}$, and since this was a practical limit for both the equipment used and for some of the other systems studied it was concluded that comparable data for all systems would be preferable. These data indicate that systems containing p-diphenylbenzene might be expected to give deposits of solid material first when operated at lower temperatures.

Plotted on the graph of Figure 9 are several points taken from data published in the literature. ${ }^{3}$ These were added in order to give a comparison with the Argonne data.

\section{Mixtures of Diphenyl with its Pyrolysis Products}

The pyrolytic still bottoms mentioned above have the analysis shown in Table IV.

Figure 11 shows melting points for this wystem, and Figure 12 gives the kinematic viscosity in centistokes for the $s$ stem as a function of composition at $130 \mathrm{C}$.

${ }^{3}$ R. J.Hood, E. E. Hardy, A. M. Ellenburg, and H. B. Richards.

"The Solubility of $\mathrm{p}-$ Terphenyl in $\mathrm{o}-$ and $\mathrm{m}-$ Terphenyls and in Diphenyl," J.Am.Chem.Soc. 75, 436, (1953) 
Table IV

APPROXIMATE ANALYSIS OF PYROLYTIC MATERIALS

Component Per cent

Dipheny1

0.8

o-terphenyl

9.9

m-terphenyl

44.7

p-terphenyl

20.4

trior thophenylene

2.0

tetraphenyls and higher

22.6

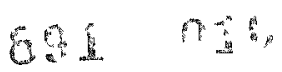




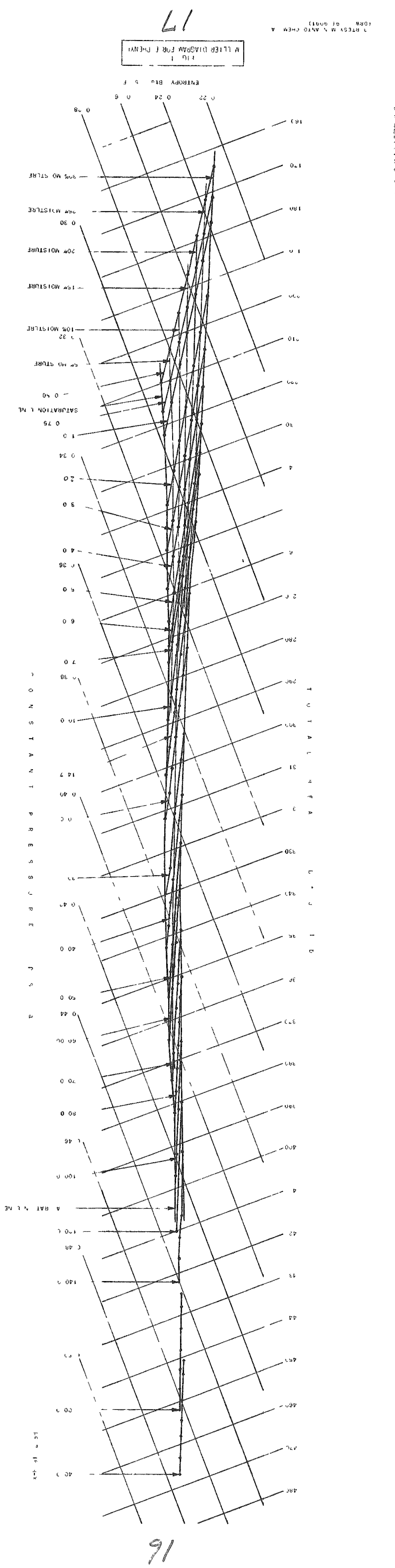




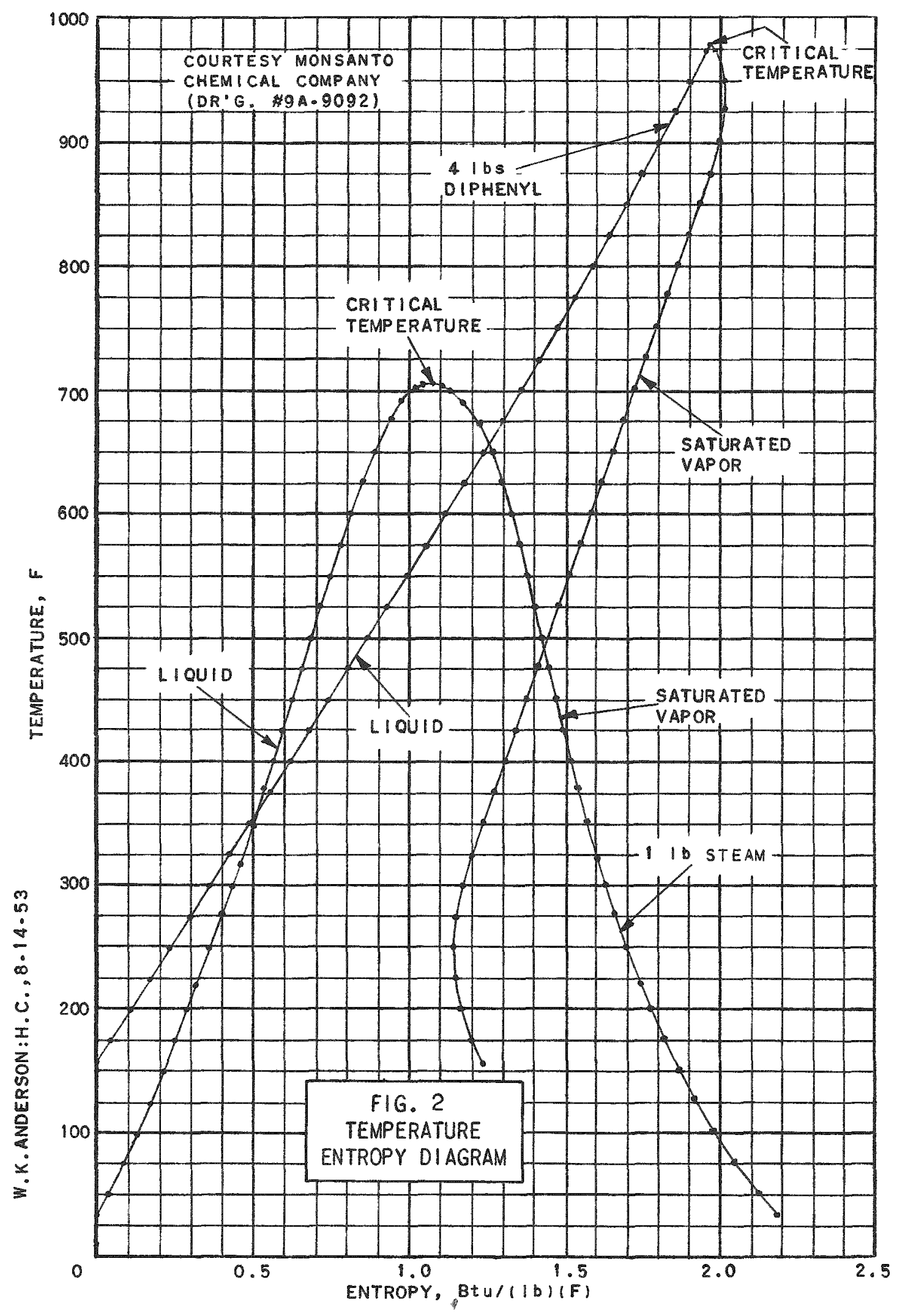

\begin{tabular}{l}
$\frac{1}{1}$ \\
$\frac{0}{0}$ \\
$\frac{1}{1}$ \\
$\frac{1}{4}$ \\
\hdashline
\end{tabular} 


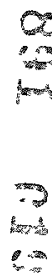

W.K. ANDERSON: F.S. 8.17 .53

pes

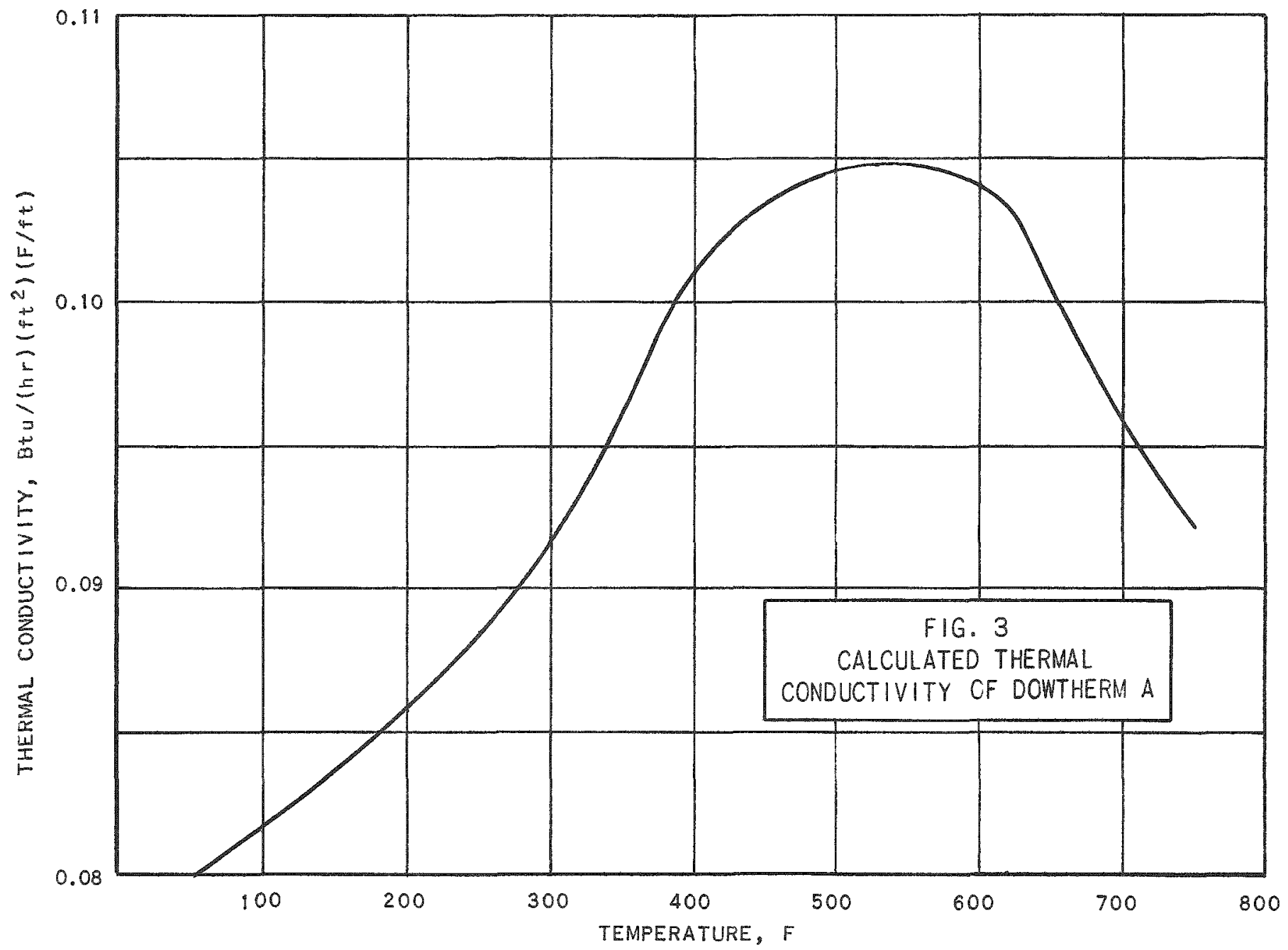

$R E-7-11505-A$ 


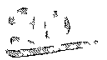

.
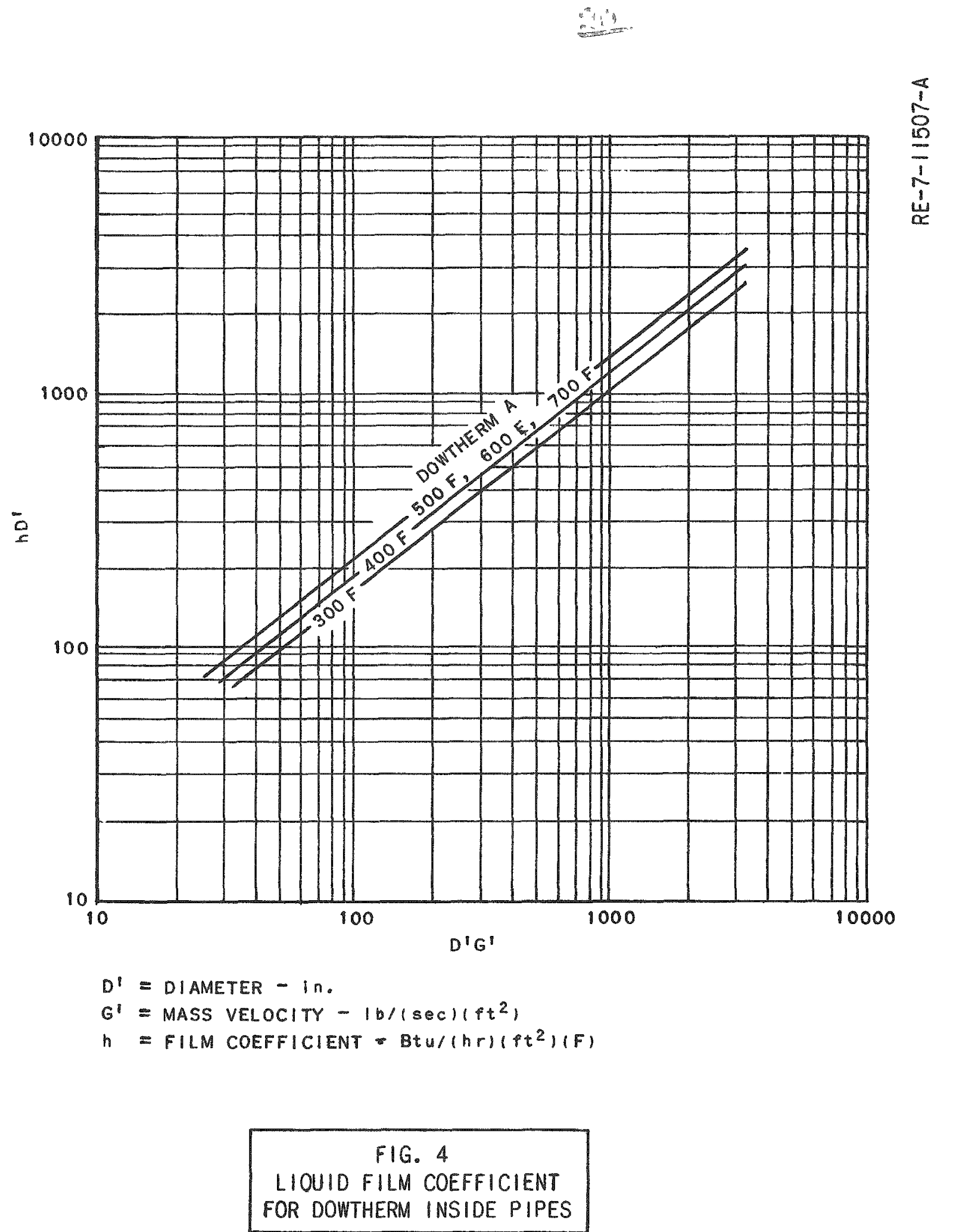

COURTESY DOW CHEMICAL CO. 


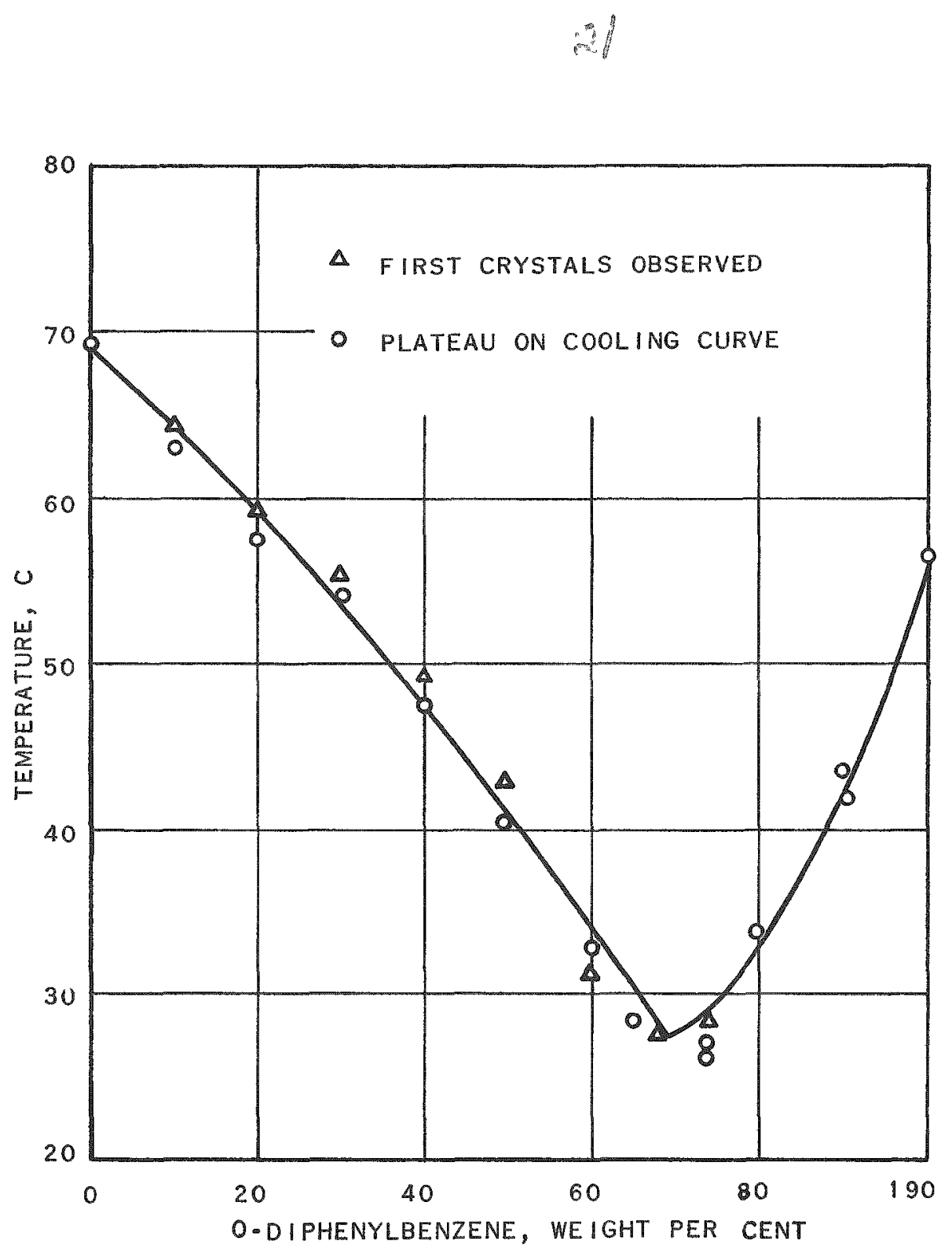

$\frac{\frac{1}{3}}{\frac{1}{2}}$

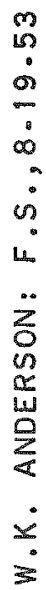

FIG. 5

MELTING POINTS

FOR THE SYSTEM

DIPHENYL - - O-DIPHENYLBENZENE 
$W . K . A N D E R S O N: F . S ., 8 \cdot 25 \cdot 53$

\section{3}

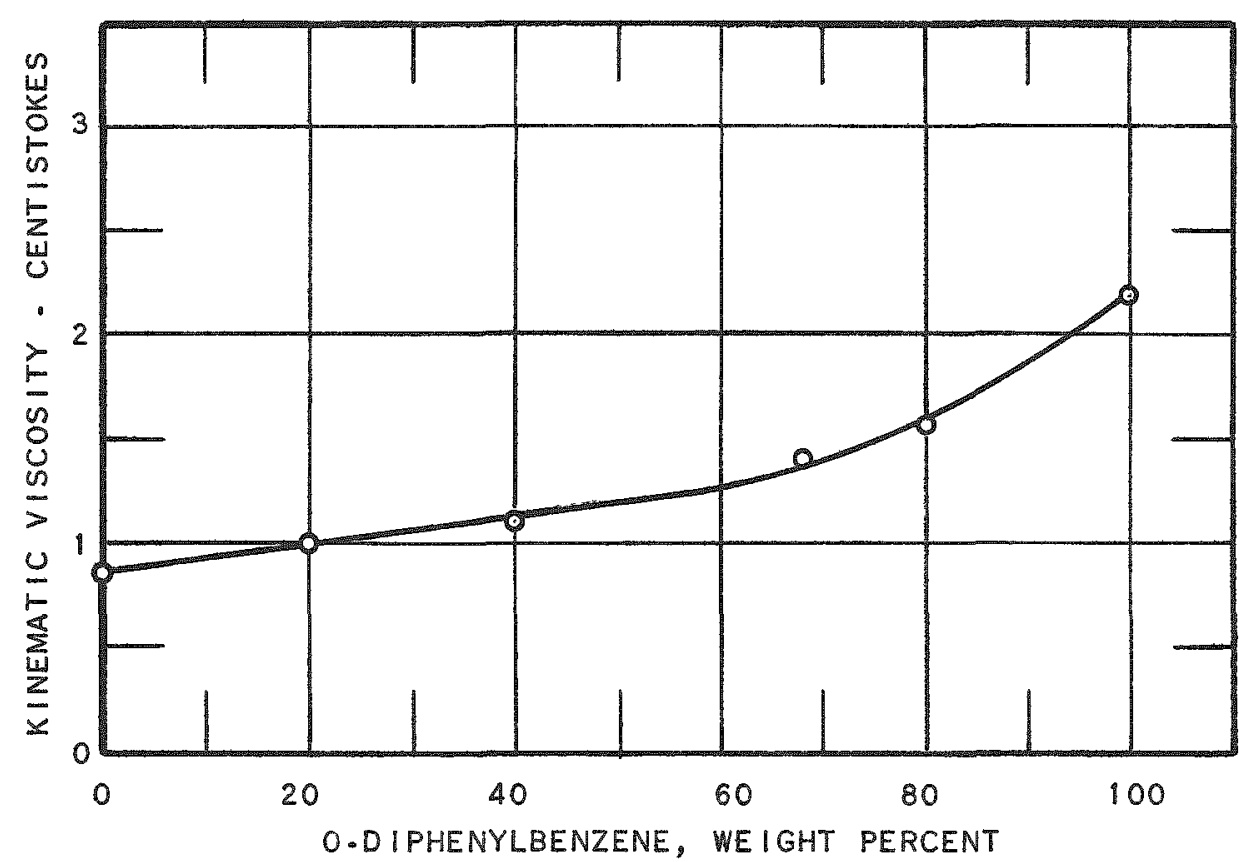

FIG. 6

KINEMATIC VISCOSITY OF MIXTURES OF DIPHENYL WITH O-DIPHENYLBENZENE AT $130 \mathrm{C}$ 


\section{2,3}

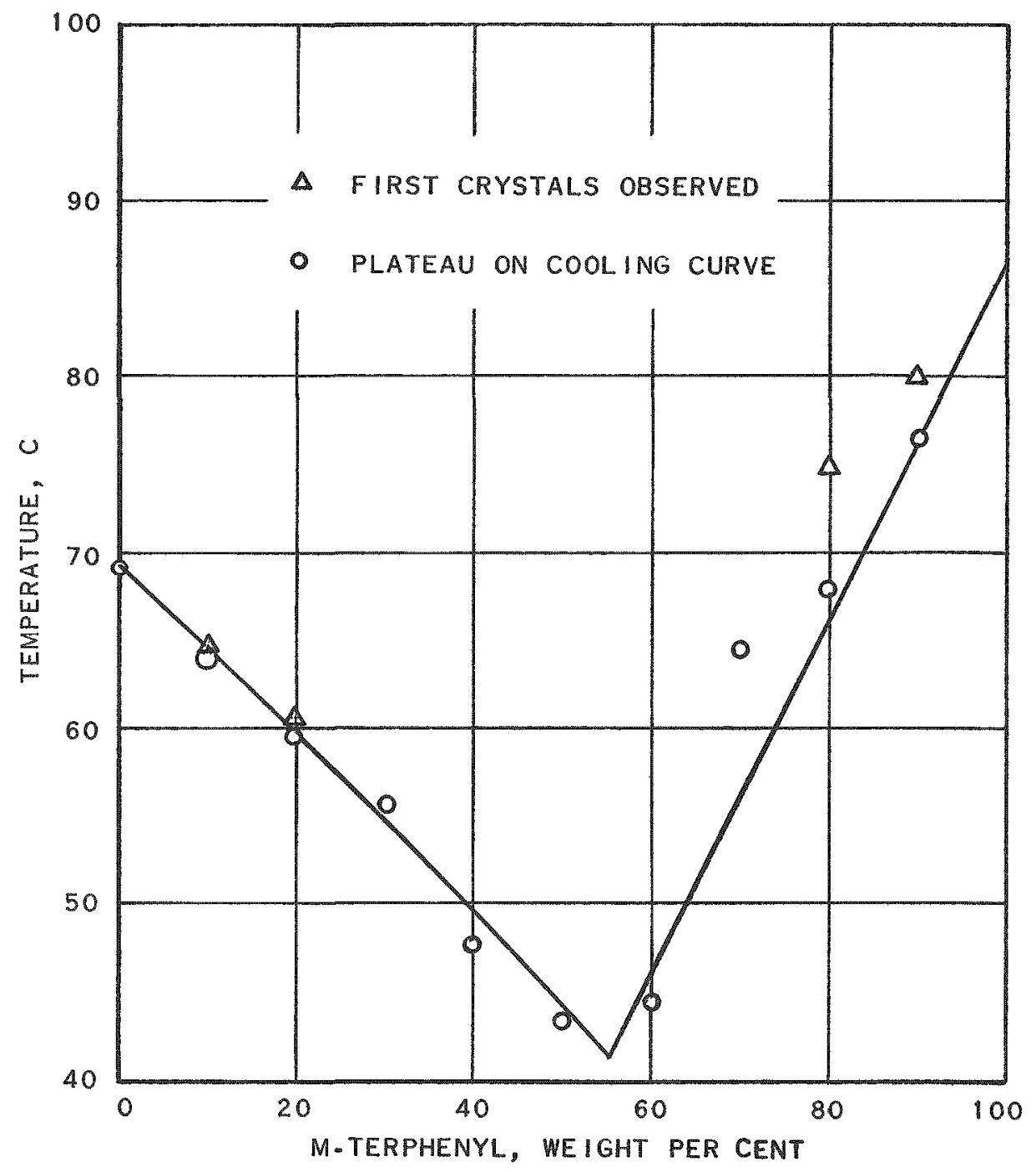

$\frac{1}{1}$
0
0
$\frac{1}{1}$
$\frac{w}{\alpha}$

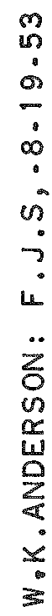

\section{FIG. 7}

MELTING POINTS

FOR THE SYSTEM

DIPHENYL - M-DIPHENYLBENZENE 
W.K.ANDERSON:F.S., $8 \cdot 25 \cdot 53$

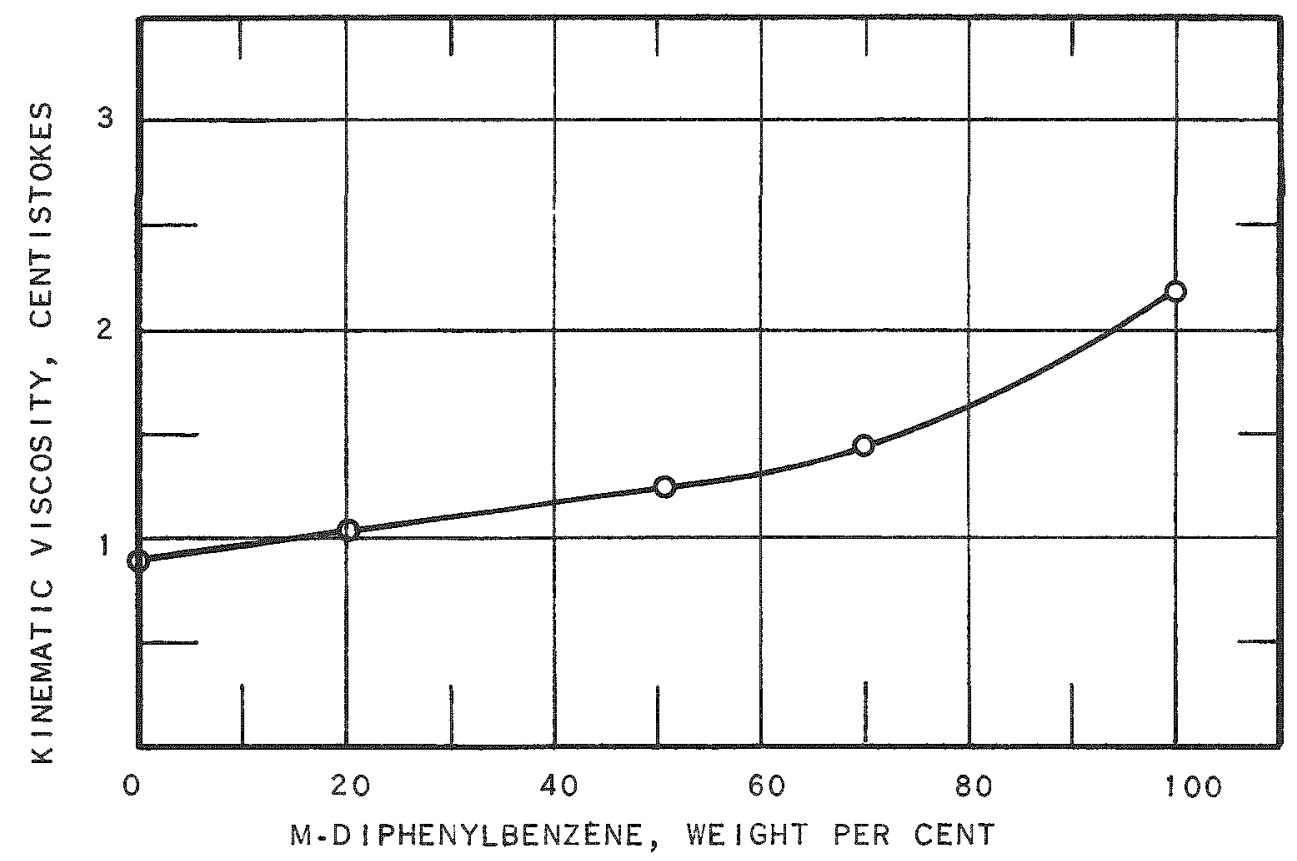

$e_{i \infty}$

FIG. 8

KINEMATIC VISCOSITY OF

MIXTURES OF DIPHENYL WITH

M-DIPHENYLBENZENE AT $130 \mathrm{C}$

3

$R E-7-11554-A$ 
is

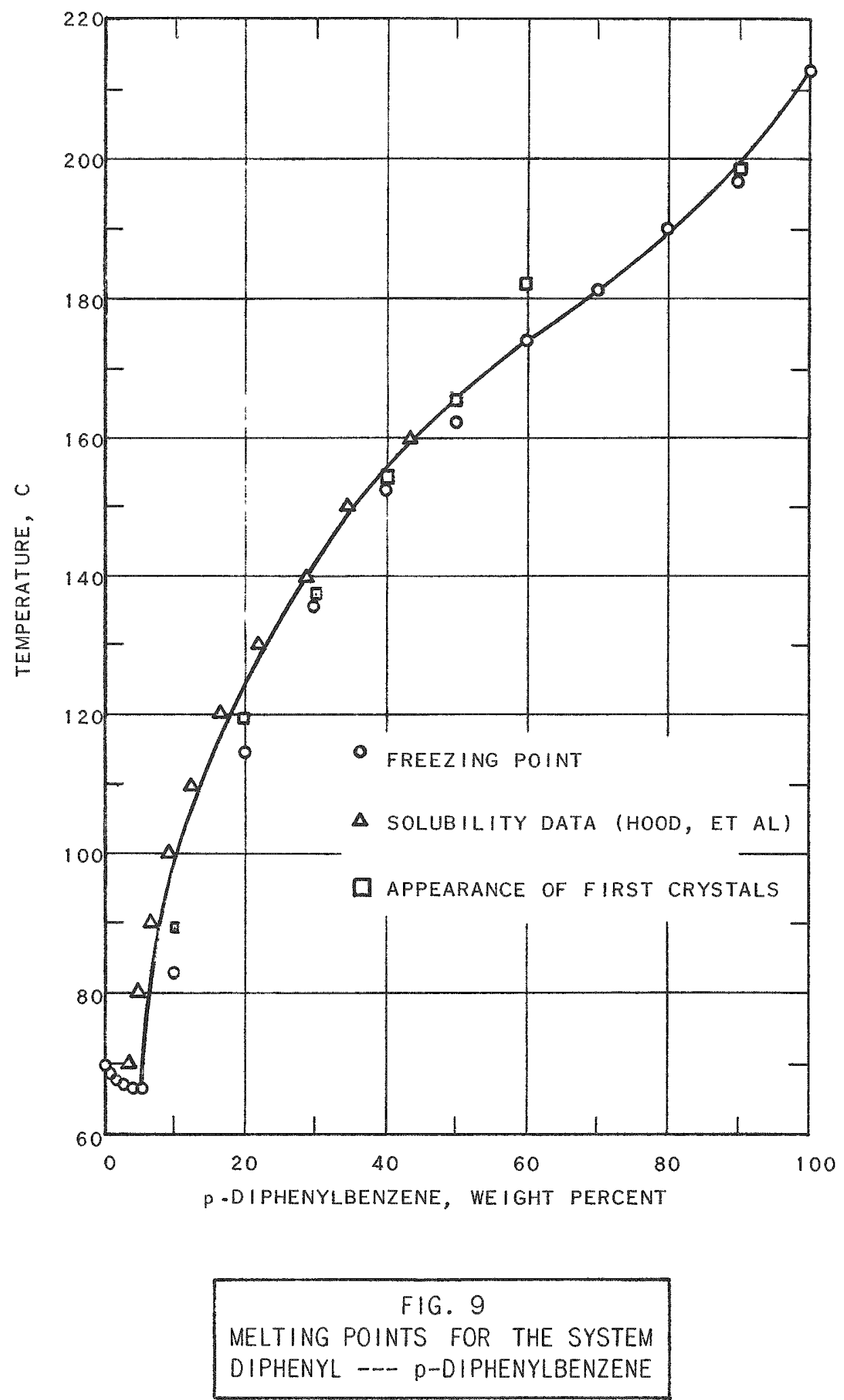

$x$
$d$
$\frac{1}{1}$
$\frac{1}{\alpha}$

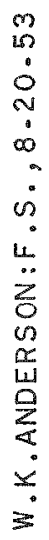


W.K.ANDERSON:F . S, $8 \cdot 25 \cdot 53$

\section{8}

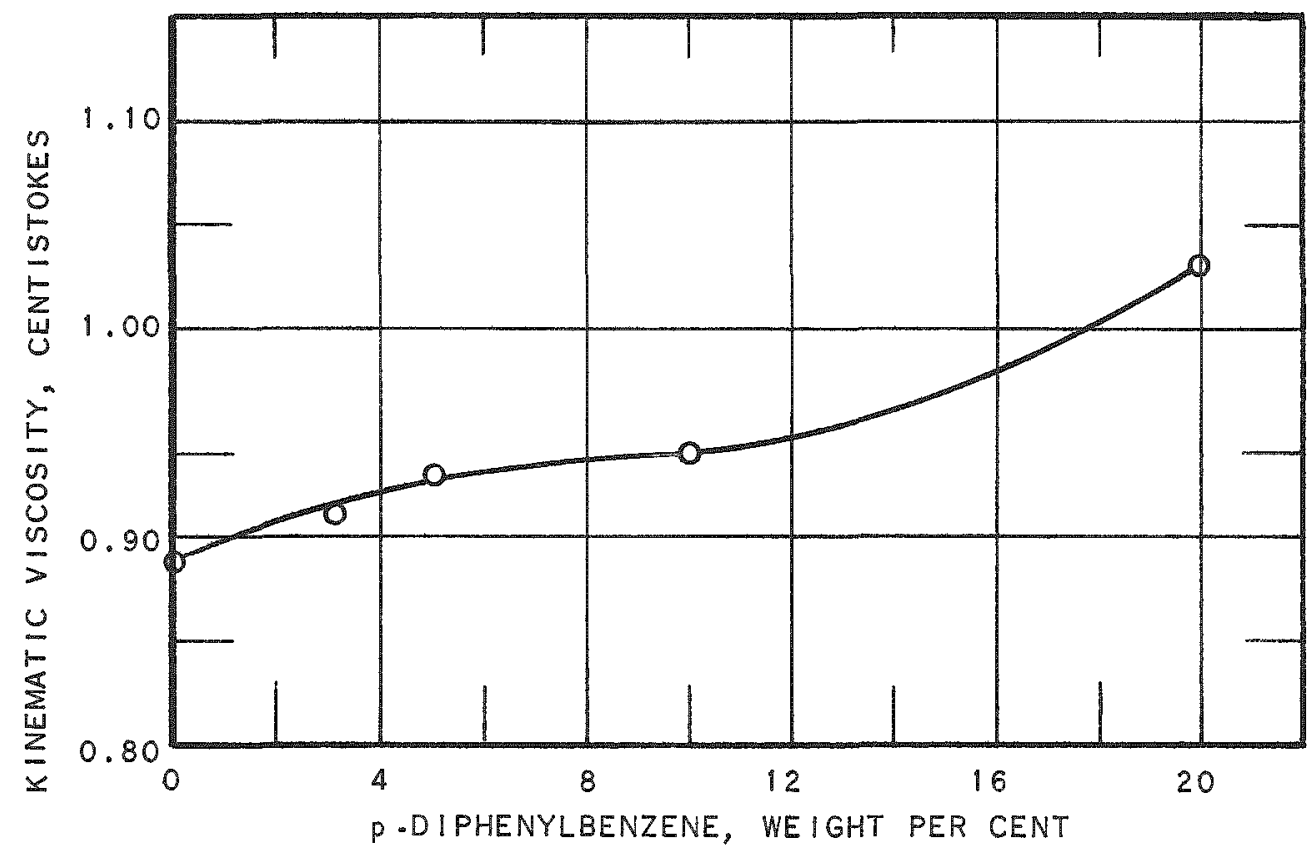

FIG. 10

KINEMATIC VISCOSITY OF MIXTURES

OF DIPHENYL WITH P-DIPHENYLBENZENE AT $130 \mathrm{C}$

$R E-7-11556-A$ 
W.K.ANDERSON : G.A.K, T/27/53

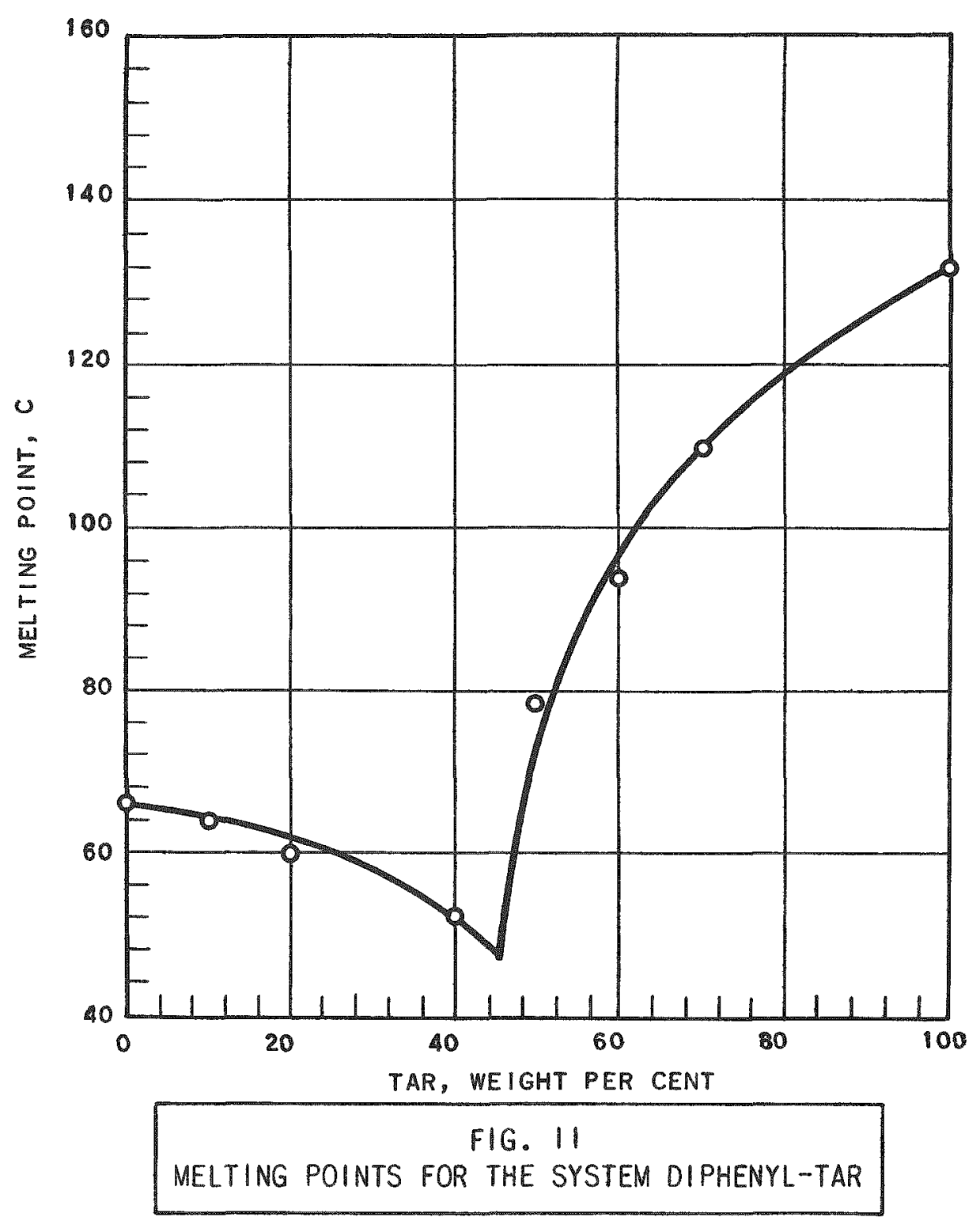

RE-7-11387-A

631 


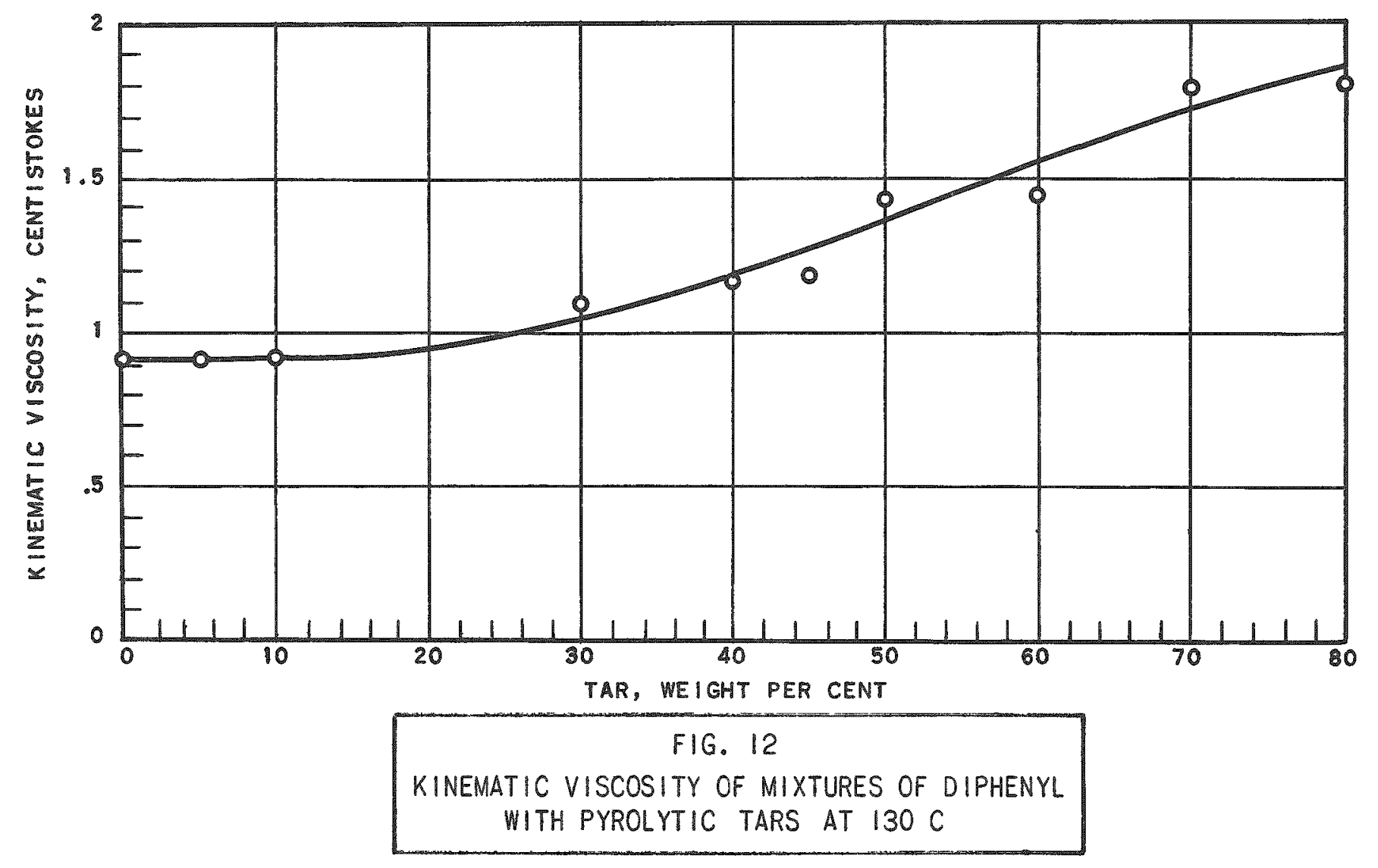

